\title{
Efficacy of tracheal tube introducers and stylets for endotracheal intubation in the prehospital setting: a systematic review and meta-analysis
}

\author{
Jaden Tollman ${ }^{1}$ (D) $\cdot$ Zubair Ahmed $^{1,2}$ (D) \\ Received: 26 April 2021 / Accepted: 27 July 2021 / Published online: 31 July 2021 \\ (c) The Author(s) 2021
}

\begin{abstract}
Purpose Tracheal tube introducers and stylets remain some of the most widely used devices for aiding practitioners in performing endotracheal intubation (ETI). The purpose of this systematic review is to evaluate the efficacy of tracheal tube introducers and stylets for ETI in the prehospital setting.

Methods A literature search was conducted on the 2nd of March 2021 across PubMed, Embase (Ovid) and the Cochrane Central Register of Controlled Trials (CENTRAL) to identify relevant studies. Included studies had their data extracted and both a quality assessment and statistical analysis were performed.

Results The summary estimate of prehospital studies with video technology showed a statistically significant increase in first pass ETI success in favour of bougies (RR 1.15, CI 1.10-1.21, $p<0.0001$ ). The summary estimates of prehospital studies without video technology and simulation studies with and without video technology showed no statistical difference between methods for first pass or overall ETI success. Some of the highest success rates were recorded by devices that incorporated video technology. Stylets lead to a shorter time to ETI while bougies were easier to use. Neither device was associated with a higher rate of ETI complications than the other.

Conclusion Both tracheal tube introducers and stylets function as efficacious aids to intubation in the prehospital environment. Where video technology is available, bougies could offer a statistically significant advantage in terms of first pass ETI success. Where video technology is unavailable, a combination of clinical scenario, practitioner expertise and personal preference might ultimately guide the choice of device.
\end{abstract}

Keywords Endotracheal intubation $\cdot$ Tracheal tube introducer $\cdot$ Bougie $\cdot$ Stylet $\cdot$ Prehospital

\section{Introduction}

Endotracheal intubation (ETI) can be a life-saving advanced airway management technique for critically ill or injured patients. It remains a technically difficult but vital skill for prehospital care providers, especially for physician-led teams that regularly encounter airway compromise $[1,2]$. It is not without risk, as significant morbidity and mortality

Jaden Tollman

JXT630@student.bham.ac.uk

1 Institute of Inflammation and Ageing, College of Medical and Dental Science, University of Birmingham, Edgbaston, Birmingham B15 2TT, UK

2 Surgical Reconstruction and Microbiology Research Centre, National Institute for Health Research, Queen Elizabeth Hospital, Birmingham B15 2TH, UK can result when ETI is performed poorly [3]. Success is unsurprisingly more common amongst experienced personnel [3], highlighting a need for devices that might improve success and reduce adverse events among less experienced personnel, or particularly difficult airways.

Tracheal tube introducers (commonly referred to as bougies) and stylets are two of the most widely used devices that aid practitioners in ETI. Bougies can be passed under full or partial vision or even blindly through the vocal cords, providing a scaffold that the endotracheal tube (ETT) can be easily passed over before the bougie is withdrawn [4]. They vary in size, shape, structure and thickness and may be single-use or reusable, angulated or straight, soft tip, hard tip or even hollow-core [4]. Stylets can be inserted into the ETT before intubation, allowing it to be moulded into a shape that facilitates easier ETI before being removed as soon as the 
cords are passed [4]. These are also manufactured in various forms similar to bougies [4].

Clinical practice varies between healthcare systems and there is no universal consensus on when and how these two devices should be implemented into airway management protocols. In the emergency department (ED), success rates for ETI can be as high as $99 \%$ with first pass rates of 80-90\% [5]. Although ETI is used in EDs to secure patient airways, a Cochrane review in 2018 found no difference between ETI and other airway securing strategies for reducing death or injury and indicated better studies were needed to make conclusive statements [6]. Both the Difficult Airway Society and American Society of Anesthesiologists currently recommend that intubating bougies and stylets may form part of a difficult airway intubation strategy [7, 8], while the Resuscitation Council UK believe a bougie should always be available for prehospital ETI [9]. A previous systematic review and meta-analysis was conducted that sought to compare the efficacy of both devices in patients undergoing ETI, though only one prehospital study was included [10]. These results may, therefore, not be generalisable to the vastly different prehospital environment.

The aim of this study was to systematically review the literature relevant to the use of bougies and stylets for ETI in the prehospital setting. This should inform a conclusion regarding their prehospital efficacy and allow practitioners to decide whether and how best to incorporate these devices into their practice. The primary outcomes of this systematic review are first pass ETI and overall ETI success. Secondary outcomes include time to ETI, ease-of-use and ETI complications such as oesophageal intubation.

\section{Methods}

\section{Literature search}

We used the search strategies recommended for the Preferred Reporting Items for Systematic Reviews and Meta-Analysis (PRISMA) statement [11]. We conducted a search on the 2nd of March 2021 across the following databases: PubMed, Embase (Ovid) and the Cochrane Central Register of Controlled Trials (CENTRAL). The search terms used were as follows: "Prehospital" AND ("Tracheal tube introducer" OR "Gum elastic bougie" OR "Flexible tip bougie" OR "Eschmann tracheal tube introducer" OR "Frova introducer" OR "Stylet"). No publication date limitations were set.

\section{Inclusion and exclusion criteria}

Studies were included if they met the following criteria: (1) clinical trials and observational studies that examine the use of laryngoscopes with tracheal tube introducers and/ or stylets by prehospital personnel in a prehospital setting/ simulation; (2) publications from any date and country; (3) publications in English; (4) human participants of all ages and simulation models of any type.

Studies were excluded on the following basis: (1) clinical trials and observational studies that examine the use of laryngoscopes with tracheal tube introducers and/or stylets by hospital personnel in a hospital setting/simulation; (2) reviews, guidelines, editorials, letters, case reports, conference abstracts and animal studies (3) publications not in English language.

\section{Data collection process}

Both authors independently performed the literature search and titles and abstracts were manually screened followed by removal of duplicates. Screened articles then underwent an independent full-text assessment for eligibility by both reviewers. The reference lists of included articles were screened again for additional studies that may have been missed. Any discrepancies were resolved by discussion between the authors.

\section{Data extraction}

We extracted the following data: (1) characteristics of included studies; (2) first pass ETI success and overall ETI success of included studies.

\section{Quality analysis}

The National Institutes of Health (NIH) quality assessment tool for observational cohort and cross-sectional studies was used independently by both reviewers (J.T., and Z.A.) to assess the methodological quality of included studies [12]. This tool assessed 14 criteria including study objectives, population, sample, exposures, outcomes and analyses. Any discrepancies were resolved by discussion between the authors.

\section{Statistical analysis}

Data were analysed using Review Manager 5.4 (The Cochrane Collaboration, Oxford, UK). Dichotomous outcomes were analysed using risk ratios (RR) as summary statistics. The effect sizes were reported as weighted mean differences and the precision of effects sizes were reported at a confidence interval (CI) of $95 \%$. A pooled estimate of RRs and weighted mean differences were computed using the DerSimonian and Laird random effects model [13]. Statistical significance was indicated by $p<0.05$ or $95 \%$ CI. Statistical heterogeneity and inconsistency in treatment effects across studies were evaluated using Cochrane $Q$ test and 
$I^{2}$ statistics, respectively. Statistical significance was set at $p<0.10$ for the Cochrane $Q$ test. Statistical heterogeneity across studies was assessed using the $I^{2}$ test (total outcome variability across studies).

\section{Results}

\section{Study selection}

Our search strategy identified a total of 68 articles across the PubMed, Embase (Ovid) and CENTRAL databases. Following removal of 22 duplicates, 46 articles were screened by title and abstract, of which 32 were excluded. The remaining 14 articles had their full-texts assessed for eligibility. Five full-text articles were excluded, two reported the use of a bougie/stylet in an exchange manoeuvre, one reported the use of a stylet without laryngoscope and two did not report bougie/stylet efficacy outcomes at all. The nine remaining studies had their reference lists screened and a further four studies were identified and included. A total of 13 studies were therefore included in our qualitative synthesis [14-26]. Of these studies, 10 were included in our quantitative synthesis $[14,15,19-26]$. This study selection process has been summarised in a PRISMA flow chart (Fig. 1).

\section{Study characteristics}

Five of the studies took place in the prehospital setting [14-16, 20, 21], while eight had a design that attempted to simulate the prehospital setting [17-19, 22-26]. Six studies took place in the United States of America (USA) [14, 18-20, 23, 26] followed by two in France [16, 21], two in Australia [22, 25], one in Finland [15], one in Turkey [17] and one in Poland [24]. Each study examined various intervention combinations of direct or video ETI assisted by different types of bougies and stylets. Studies reported a variety of relevant outcomes including first pass ETI success, overall ETI success, time to ETI, ease-of-use and ETI complications. A full overview of the characteristics of included studies is given in Table 1 .

\section{Quality analysis}

Figure 2 summarises the results from our quality analysis of included studies using the NIH quality assessment tool for observational cohort and cross-sectional studies. For each study, a number of "yes", "no", or "cannot determine (CD)/not applicable (NA)/not reported (NR)" answers were assigned depending on whether each of the tool's 14 criteria were satisfied. None of the studies were assigned an answer of "yes" for all 14 criteria. Bonnette et al. [14] had the highest proportion (79\%) of criteria answered "yes" (i.e. high quality) while Ångerman et al. [15] and Messa et al. [23] had the lowest proportion (57\%) answered "yes". Ångerman et al. [15] also had the highest proportion (36\%) of criteria answered "no" while Bonnette et al. [14], Cooney et al. [19], Evrin et al. [24] and Gregory et al. [25] all had the lowest proportion (14\%) answered "no". Every study had at least $7 \%$ of criteria answered "CD/NA/NR" as every study investigated an exposure that could not vary in amount or level. Eight studies had 14\% of criteria answered "CD/NA/ NR" [17-19, 22-26] as it could not be determined whether the participation rate of eligible persons was at least $50 \%$.

\section{Review of primary outcomes}

Table 2 summarises the primary outcomes of first pass ETI success and overall ETI success for studies with a prehospital design without video technology [16, 20, 21]. Jabre et al. [16] was the only study that did not fully report on first pass ETI success. The highest first pass ETI success rate was reported by Combes et al. [21] at 74\% when a non-bougie-assisted direct ETI method was implemented. Combes et al. [21] also reported the lowest first past ETI success rate at just $2 \%$ when a bougie-assisted direct ETI method was used. Neither Jabre et al. [16] nor Heegaard et al. [20] fully reported on overall ETI success. Combes et al. [21] reported the highest overall ETI success rate with their non-bougieassisted direct ETI method at $90.3 \%$. The lowest overall ETI success rate of $75.5 \%$ was again reported by Combes et al. [21] using a bougie-assisted method of direct ETI.

Table 3 summarises the primary outcomes of first pass ETI success and overall ETI success for studies with a prehospital design with video technology $[14,15]$. Both studies fully reported on first pass ETI success. Ångerman et al. [15] reported the highest first pass ETI success rate of $98.2 \%$ when their new protocol of bougie-assisted video ETI was implemented. While Bonnette et al. [14] reported the lowest first pass ETI success rate of $43.8 \%$ using a nonbougie-assisted direct/video ETI method. Both studies also fully reported on overall ETI success. Ångerman et al. [15] reported the highest overall ETI success rate at $99.6 \%$ using their new protocol of bougie-assisted video ETI. Again, Bonnette et al. [14] reported the lowest overall ETI success rate of $49.1 \%$ using a method of non-bougie-assisted direct/ video ETI.

Table 4 summarises the primary outcomes of first pass ETI success and overall ETI success for studies with a simulation design without video technology [17, 18, 23-26]. Only Evrin et al. [24] fully reported on first pass ETI success, with a highest rate of $91.3 \%$ for their new model bougieassisted direct ETI method and lowest rate of $23.9 \%$ for their non-bougie-assisted direct ETI method. All six studies fully reported on overall ETI success. The highest overall ETI success rates of $100 \%$ were reported by Karaca et al. [17] 


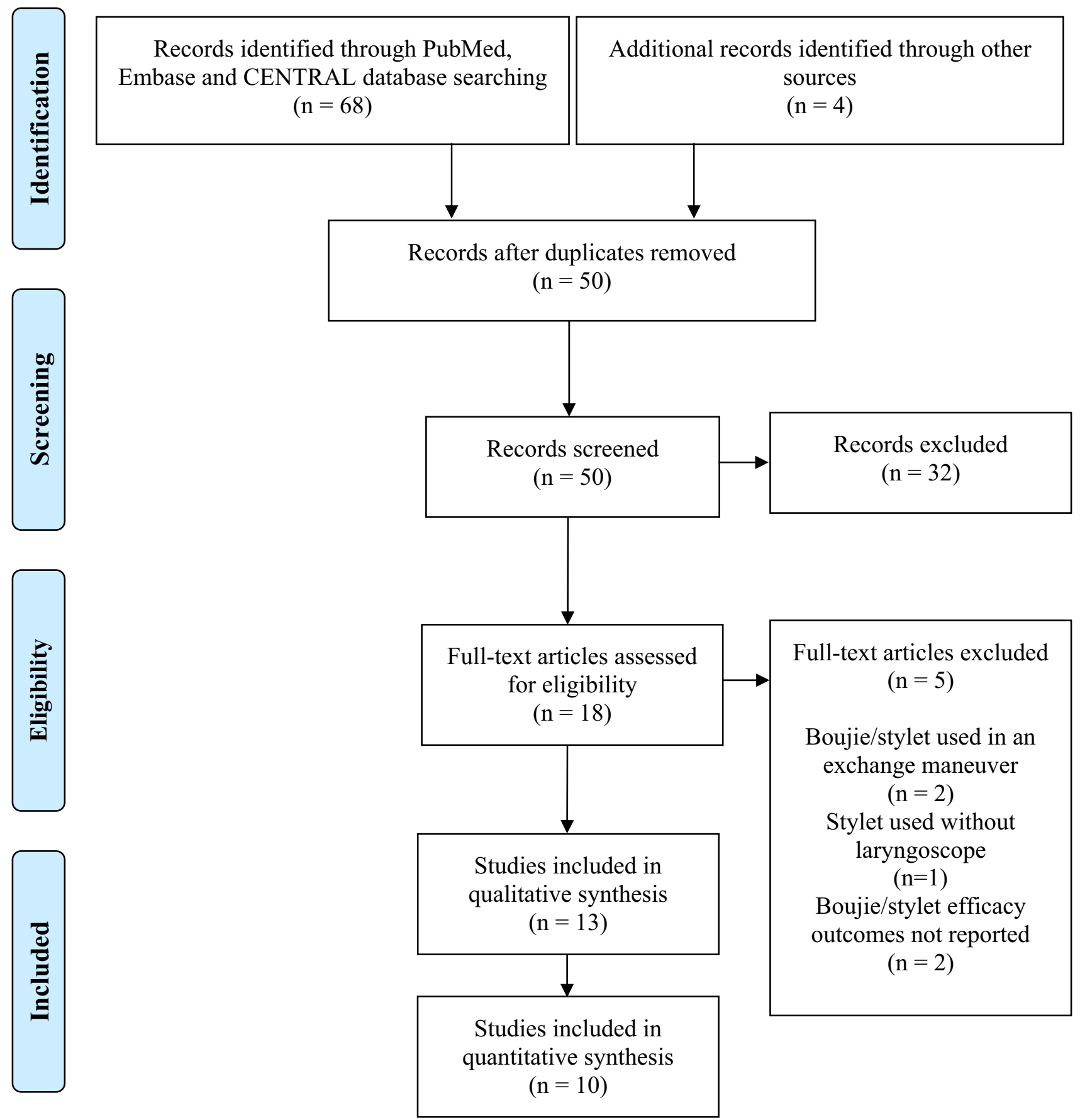

Fig. 1 PRISMA flowchart summarising study selection process

for both their moving and stationary bougie-assisted direct ETI methods, and Evrin et al. [24] for both their standard and new model bougie-assisted direct ETI methods. Gregory et al. [25] reported the lowest overall ETI success rate at just $8 \%$ when using a Portex (reusable) bougie for direct ETI.

Table 5 summarises the primary outcomes of first pass ETI success and overall ETI success for studies with a simulation design with video technology [19, 22]. Both studies fully reported on first pass ETI success. Cooney et al. [19] reported the highest first pass ETI success rate of $96.3 \%$ using a method of video-stylet-assisted direct ETI. While the lowest first pass ETI success rate of $0 \%$ was reported by Woollard et al. [22]. Both studies also fully reported on overall ETI success. Again, Cooney et al. [19] reported the highest overall ETI success rate of $100 \%$ using a method of stylet-assisted direct ETI. While Woollard et al. [22] 


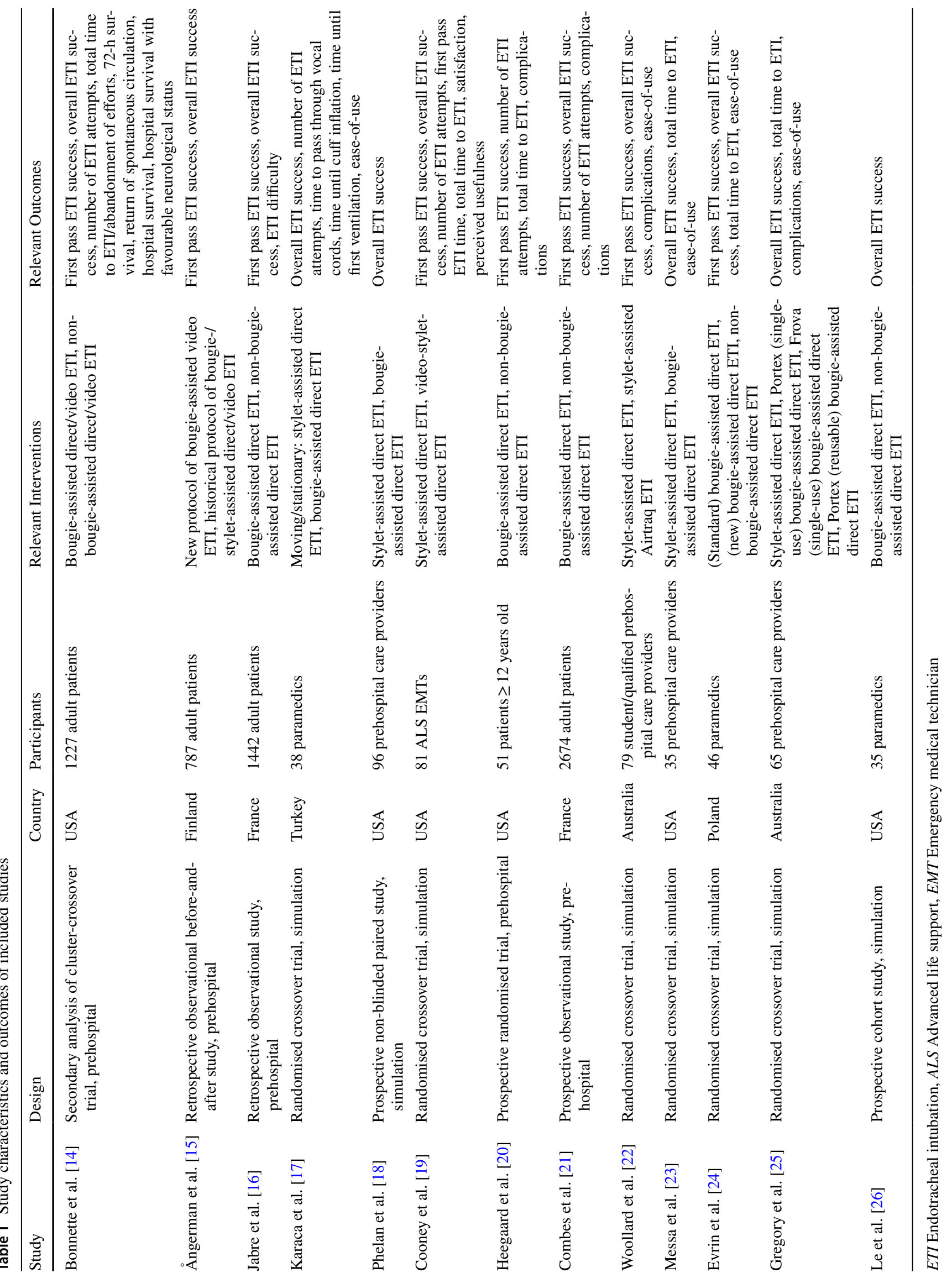


Fig. 2 Summary of NIH quality assessment tool for observational cohort and cross-sectional studies results

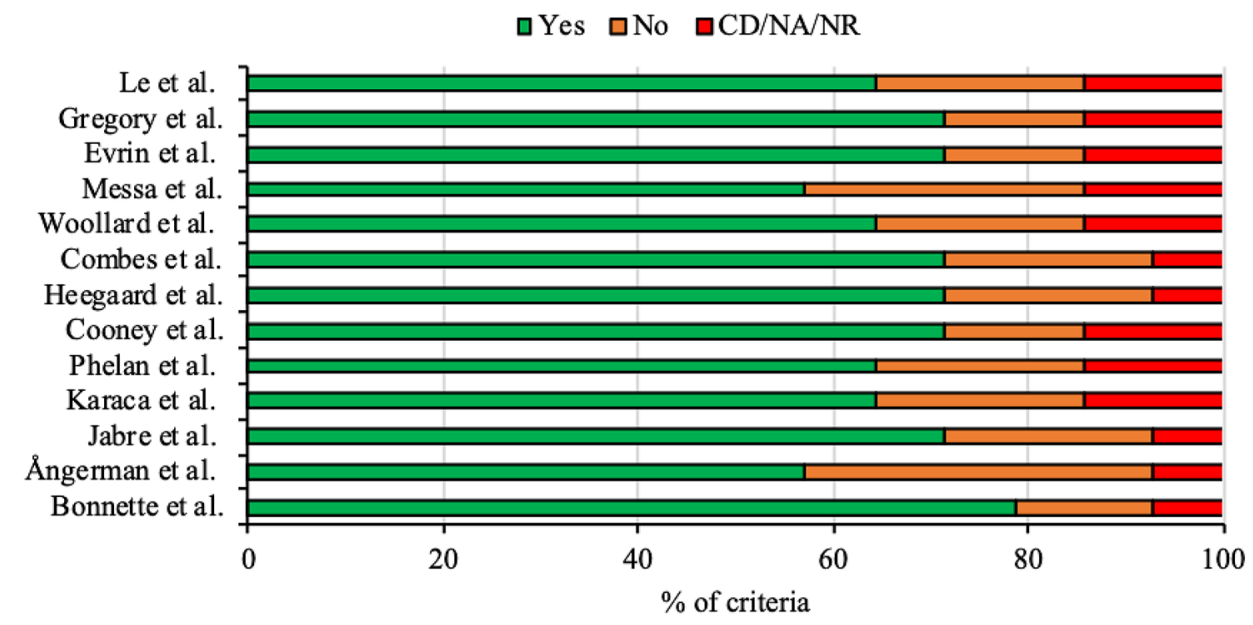

Table 2 First pass ETI success and overall ETI success of prehospital studies without video technology

\begin{tabular}{lllll}
\hline Study & First pass ETI success (\%) & Overall ETI success (\%) & \\
\hline Jabre et al. [16] & Bougie-assisted direct ETI & Non-bougie-assisted direct ETI & Bougie-assisted direct ETI & Non-bougie-assisted direct ETI \\
& 58.5 & Not reported & 80.5 & Not reported \\
Heegaard et al. [20] & Bougie-assisted direct ETI & Non-bougie-assisted direct ETI & Bougie-assisted direct ETI & Non-bougie-assisted direct ETI \\
& 70.0 & 65.0 & Not reported & Not reported \\
Combes et al. [21] & Bougie-assisted direct ETI & Non-bougie-assisted direct ETI & Bougie-assisted direct ETI & Non-bougie-assisted direct ETI \\
& 2.0 & 74.0 & 75.5 & 90.3 \\
\hline
\end{tabular}

ETI Endotracheal intubation

Table 3 First pass ETI success and overall ETI success of prehospital studies with video technology

\begin{tabular}{|c|c|c|c|c|}
\hline \multirow{2}{*}{$\frac{\text { Study }}{\text { Bonnette et al. [14] }}$} & \multicolumn{2}{|c|}{ First pass ETI success (\%) } & \multicolumn{2}{|c|}{ Overall ETI success (\%) } \\
\hline & $\begin{array}{l}\text { Bougie-assisted } \\
\text { direct/video } \\
\text { ETI }\end{array}$ & Non-bougie-assisted direct/video ETI & $\begin{array}{l}\text { Bougie-assisted } \\
\text { direct/video } \\
\text { ETI }\end{array}$ & Non-bougie-assisted direct/video ETI \\
\hline & 52.1 & 43.8 & 56.1 & 49.1 \\
\hline \multirow[t]{2}{*}{ Ångerman et al. [15] } & $\begin{array}{l}\text { New protocol of } \\
\text { bougie-assisted } \\
\text { video ETI }\end{array}$ & $\begin{array}{l}\text { Historical protocol of bougie-/stylet- } \\
\text { assisted direct/video ETI }\end{array}$ & $\begin{array}{l}\text { New protocol of } \\
\text { bougie-assisted } \\
\text { video ETI }\end{array}$ & $\begin{array}{l}\text { Historical protocol of bougie-/stylet- } \\
\text { assisted direct/video ETI }\end{array}$ \\
\hline & 98.2 & 85.7 & 99.6 & 99.2 \\
\hline
\end{tabular}

ETI Endotracheal intubation

reported the lowest overall ETI success rate of 30\% using their method of stylet-assisted direct ETI.

Two studies [20, 21] were included in our meta-analysis of bougie versus stylet first pass ETI success for prehospital studies without video technology (Fig. 3) The primary outcome of first pass ETI success was chosen for this analysis as overall ETI success was not fully reported in two of the prehospital studies without video technology [16, 20]. Statistical heterogeneity across studies was high $\left(I^{2}=98 \%\right)$. The results of Combes et al. [21] favoured the use of a stylet (RR 0.17, CI 0.12-0.24), while Heegard et al. [20] did not show a statistically significant difference between devices
(RR 1.08, CI 0.74-1.60). Overall, the summary estimate of prehospital studies without video technology showed no statistically significant difference between devices for first pass ETI success (RR 0.42, CI 0.07-2.68, $p=0.36$ ).

Two studies $[14,15]$ were included in our meta-analysis of bougie versus stylet first pass ETI success for prehospital studies with video technology (Fig. 4). Statistical heterogeneity across studies was low $\left(I^{2}=0 \%\right)$. The results of Bonnette et al. [14] favoured the use of a bougie (RR 1.19, CI 1.05-1.34), while Ångerman et al. [15] did not show a statistically significant difference between devices (RR 1.15, CI 1.09-1.21). Overall, the summary estimate of prehospital 


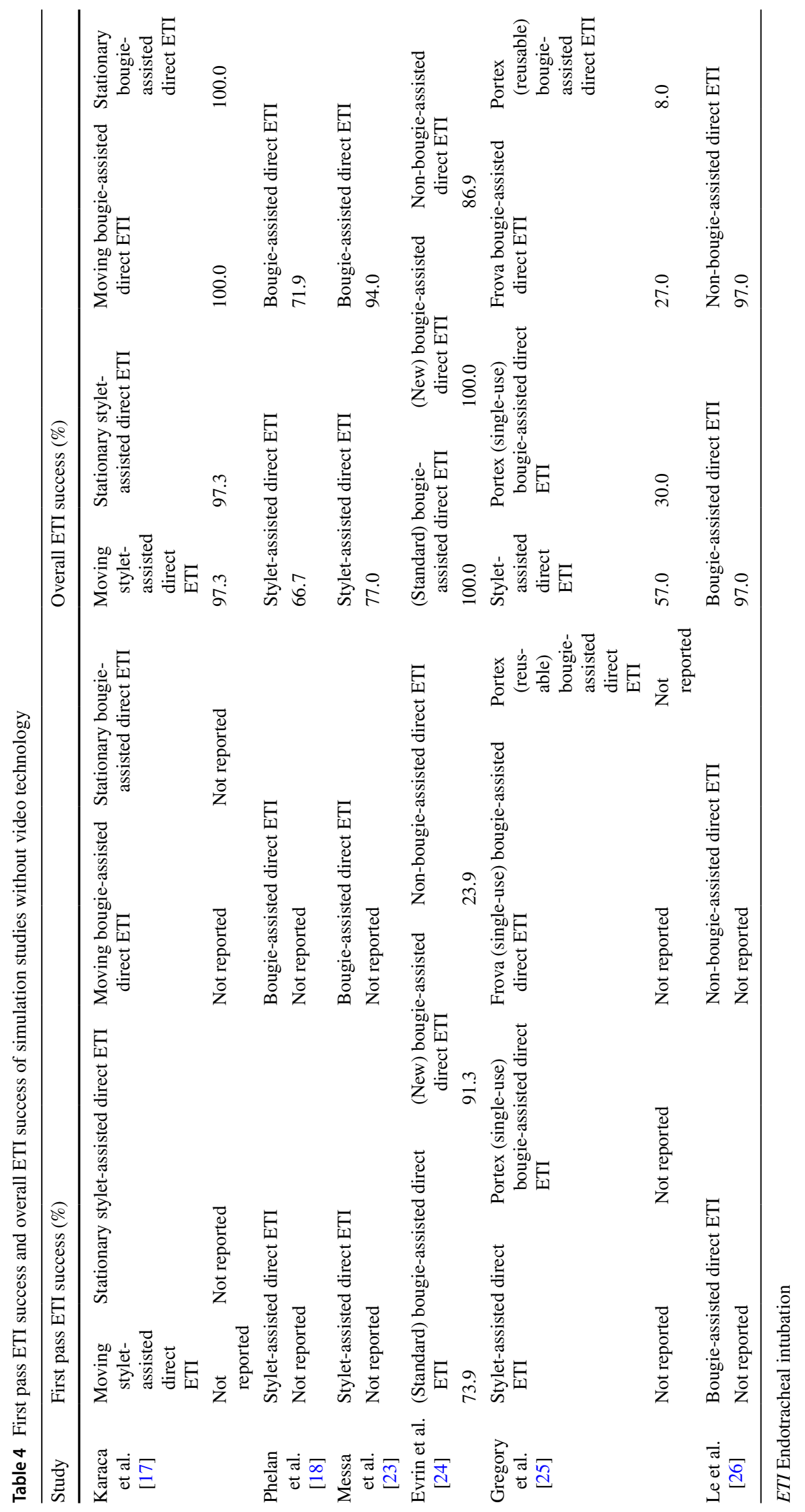


Table 5 First pass ETI success and overall ETI success of simulation studies with video technology

\begin{tabular}{llllc}
\hline Study & First pass ETI success (\%) & \multicolumn{2}{c}{ Overall ETI success (\%) } & \\
\hline Cooney et al. [19] & Stylet-assisted direct ETI & $\begin{array}{c}\text { Video-sty- } \\
\text { let-assisted } \\
\text { direct ETI }\end{array}$ & Stylet-assisted direct ETI & $\begin{array}{c}\text { Video- } \\
\text { stylet- } \\
\text { assisted } \\
\text { direct }\end{array}$ \\
& & & & ETI \\
& 95.1 & 96.3 & 100.0 & 98.8 \\
Woollard et al. [22] & Stylet-assisted direct ETI & Stylet- & Stylet-assisted direct ETI & Stylet- \\
& & assisted & Airtraq \\
& & ETI & & Airtraq \\
& & 44.0 & 30.0 & ETI \\
& 0.0 & & & 78.0 \\
\hline
\end{tabular}

ETI Endotracheal intubation

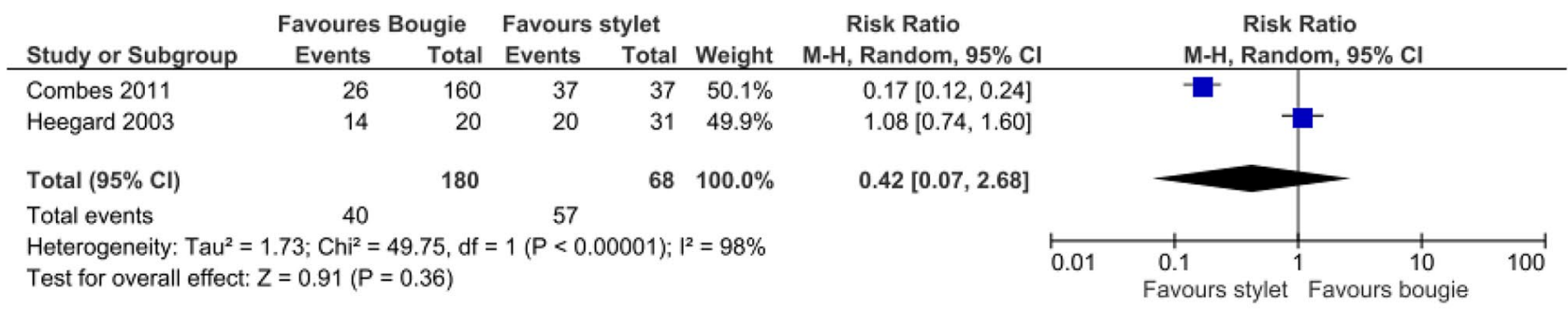

Fig. 3 Forest plot of comparison between bougie versus stylet; outcome, first pass success rate in studies without video technology

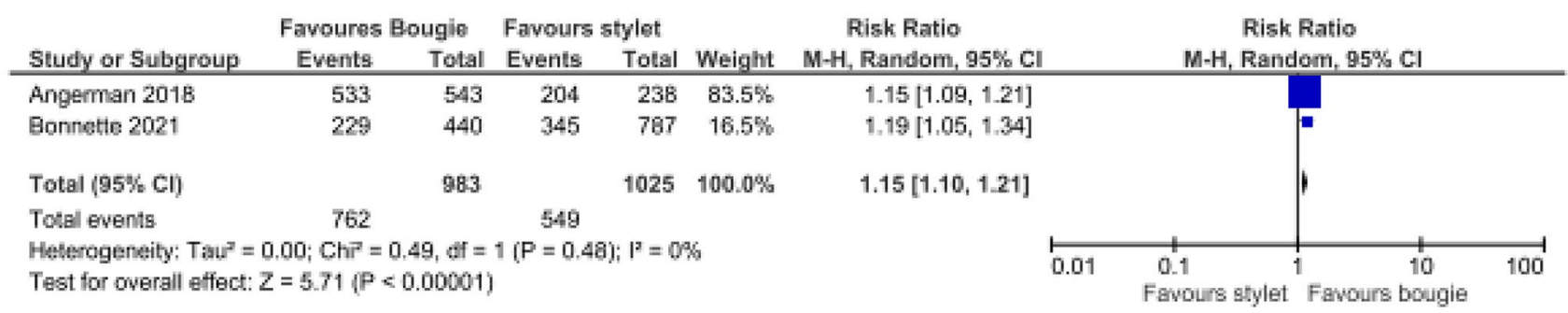

Fig. 4 Forest plot of comparison between bougie versus stylet; outcome, first pass success rate in studies with video technology

studies with video technology showed a statistically significant difference between devices for first pass ETI success, favouring the use of a bougie (RR 1.15, CI 1.10-1.21, $p<0.00001)$.

Four studies [23-26] were included in our meta-analysis of a bougie versus non-bougie method overall ETI success for simulation studies without video technology (Fig. 5). The primary outcome of overall ETI success was chosen for this analysis as first pass ETI success was not fully reported in five of the simulation studies without video technology [17, $18,23,25,26]$. Statistical heterogeneity across studies was high $\left(I^{2}=83 \%\right)$. All four studies [23-26] showed no statistical difference between ETI methods. Overall, the summary estimate of simulation studies without video technology showed no statistical difference between methods for overall ETI success (RR 1.06, CI 0.93-1.21, $p=0.39$ ).
Two studies [19,22] were included in our meta-analysis of bougie versus non-bougie method overall ETI success for simulation studies with video technology (Fig. 6). Statistical heterogeneity across studies was again high $\left(I^{2}=98 \%\right)$. The results of Woollard et al. [22] favoured the use of a bougie (RR 2.57, CI 1.34-4.95), while Cooney et al. [19] showed no statistical difference between methods (RR 0.99, CI 0.95-1.02). Overall, the summary estimate of simulation studies with video technology showed no statistical difference between methods for overall ETI success (RR 1.58, CI $0.14-17.34, p=0.71$ ).

\section{Review of secondary outcomes}

Seven studies reported on time to ETI $[14,17,19,20$, 23-25]. Bonnette et al. [14] reported statistically significant 


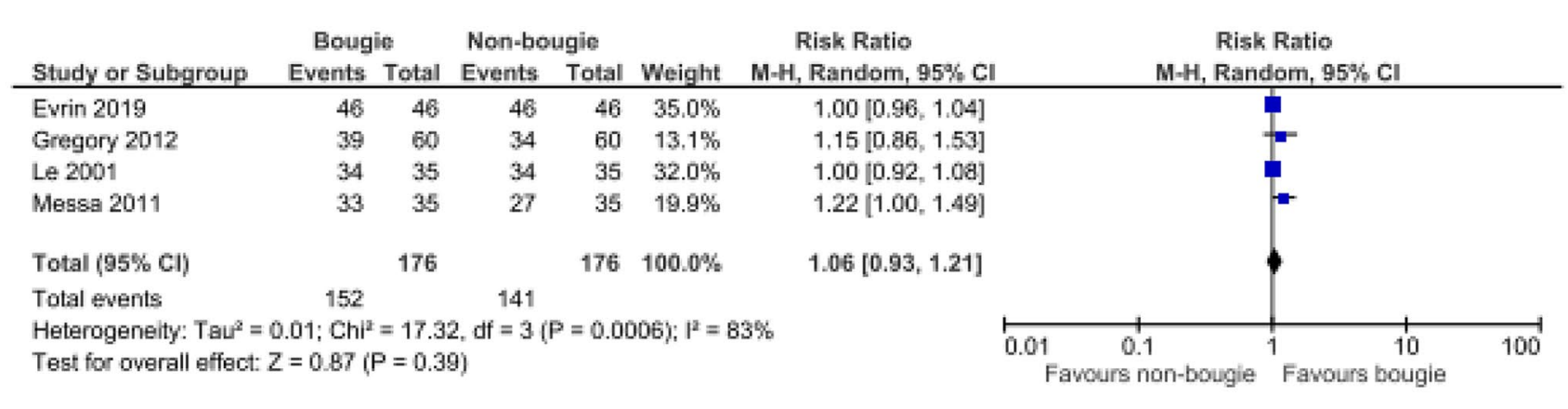

Fig. 5 Forest plot of comparison between bougie versus stylet; outcome, overall success rate in studies without video technology

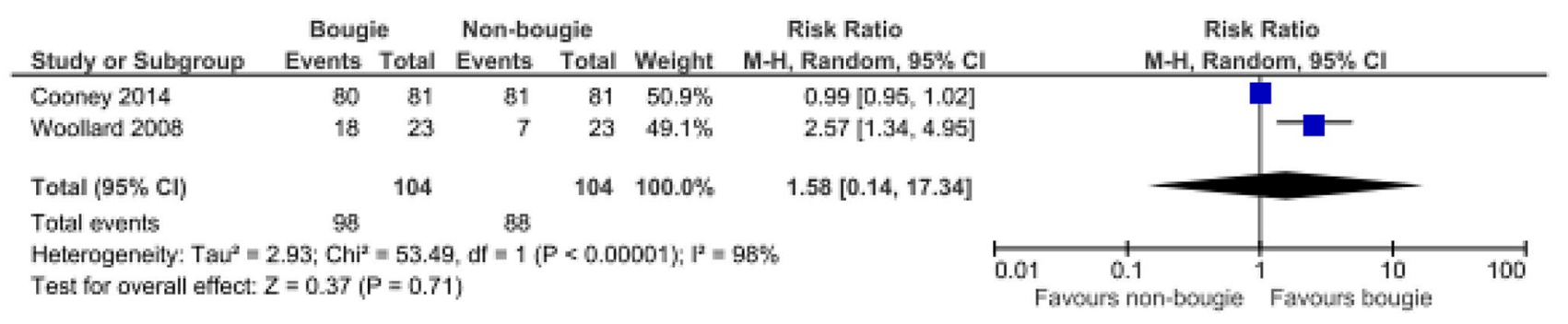

Fig. 6 Forest plot of comparison between bougie versus stylet; outcome, overall success rate in studies with video technology

results that using a bougie with either direct or videolaryngoscopy led to a longer time to ETI compared to a stylet [13.0 min vs $11.0 \mathrm{~min}$, HR 0.63 , 95\% CI $0.45-0.90$ ). Karaca et al. [17] supports this, noting a significantly shorter duration with a stylet to pass the vocal cords in a stationary ambulance, to inflate the ETT cuff in both stationary and moving ambulances and to first ventilate in a moving ambulance. Gregory et al. [25] also concurs, reporting a significantly shorter time to ETI with a stylet compared to all three assessed bougies, none of which had a statistically significant difference in time to ETI between them. Conversely, Evrin et al. [24] was the only study that reported statistically significant results that bougies led to a shorter median intubation time, with the shortest time offered by the new model of bougie at 29 s (IQR: 25-38). Both Heegard et al. [20] and Messa et al. [23] reported no statistically significant differences in average or median time to ETI between stylets and bougies. Cooney et al. [19] noted no significant difference in median time to ETI between classic and video stylets.

Five studies reported on ease-of-use [17, 22-25]. Messa et al. [23] used a Likert ease-of-use survey and reported that overall, a significant majority of participants perceived a bougie to be easier to use than a stylet. Similarly, Evrin et al. [24] used an ease-of-use visual analogue scale (VAS) and reported that participants found bougies to be easier to use, with new model bougies being easier than standard model bougies. However, Gregory et al. [25] also used an easeof-use VAS and noted that participants found the Portex reusable bougie to be the most difficult to use of all devices. Though the other two bougies and stylet being assessed did not vary in difficulty [25]. Karaca et al. [17] was the only study to report no significant differences in perceived ETI difficulty using a bougie versus a stylet in both a moving and stationary ambulance. Comparing stylets alone, Woollard et al. [22] used an ease-of-use VAS and reported both students and practitioners found using a stylet with an Airtraq laryngoscope for ETI to be significantly easier than using a stylet with a standard Macintosh laryngoscope.

Four studies reported on ETI complications [20-22, 25 ] with oesophageal intubation being the most frequently reported. Combes et al. [21] recorded a 52\% rate of early intubation-related complications when a bougie was used for ETI, though they did not report a rate for when a bougie was not used. Oesophageal intubation accounted for the largest proportion of these complications at $36 \%$, followed by arterial oxygen desaturation at $26 \%$ [21]. Gregory et al. [25] concluded that use of the Portex reusable bougie in particular was associated with the highest risk of oesophageal placement. The other two bougies and stylet that were being assessed all had a similar risk to one another [25]. However, Heegard et al. [20] did not report a significant difference in oesophageal intubation rate between a bougie $(5 \%)$ and nonbougie $(6.5 \%)$ method of ETI. When just a stylet is used, Woollard et al. [22] reported a lower rate of oesophageal intubations when used alongside an Airtraq laryngoscope as opposed to a standard Macintosh laryngoscope. This 
was true for both students and experienced practitioners, whereby practitioners recorded zero oesophageal intubations with the Airtraq and stylet combination.

\section{Discussion}

Our systematic review qualitatively and quantitatively integrates the literature relevant to the use of bougies and stylets for ETI in the prehospital setting. It is clear that both bougies and stylets should have a role in prehospital ETI, though bougies appear to offer a statistically significant increase in first pass ETI success when used alongside video technology. Additionally, there are suggestions that particular devices may convey advantages in certain clinical situations. These devices, as well as prehospital environment considerations, practitioner experience and study design quality all necessitate consideration when choosing between bougies or stylets.

Our results suggest that bougies offer a statistically significant increase in first pass ETI success when used alongside video technology in the prehospital setting. Though, conclusions inferred from this isolated statistically significant result must be considered in the context of high study heterogeneity and the multiplicity of comparisons being made. Nevertheless, some of the highest first pass and overall ETI success rates in both prehospital and simulation studies incorporated some form of video technology. Ångerman et al. [15] achieved this in the prehospital setting by utilising a Frova intubating introducer alongside a C-MAC videolaryngoscope. Alternatively, Cooney et al. [19] achieved their high first pass success in the simulation setting by utilising the Clarus Video System (CVS), a video-assisted semi-rigid fibreoptic stylet, alongside direct laryngoscopy. Improvements in ETI success with videolaryngoscopy are not a novel concept and have been previously demonstrated by another systematic review in the hospital environment [27]. The advantage that video-assistance conveys might be more notable and prove particularly useful with novice intubators [28]. Newer technologies such as the CVS then widen the scope of how we might install video technology into pre-existing devices such as the simple malleable stylet. When working with these devices, however, consideration must be given towards the cost that is inevitably higher than in their technologically sparse counterparts. Still, whether incorporated into the analogous laryngoscope or the stylet/ bougie itself, our review highlights that video technology might offer the key to further improving upon ETI success in the prehospital setting. In particular, where video technology is available, our findings suggest that some practitioners may wish to opt for a bougie over a stylet to maximise their first pass ETI success.
Our review of secondary outcomes suggests that stylets offer speed while bougies offer ease-of-use, though neither device appears to lead to a higher rate of complications than the other. Again, conclusions inferred from these results must be considered in the context of high study heterogeneity and the multiplicity of comparisons being made. Still, where video technology is unavailable, bougies may have a role where the intubating practitioner is less experienced, while stylets may have a role in cases where catastrophic airway compromise is imminent. However, it is worth noting the results of Gregory et al. [25] reported that the Portex reusable bougie was both the most difficult to use and led to the most complications while having the lowest overall ETI success across simulation studies. A practitioner's specific preference of bougie might therefore instead be towards the Portex single-use or Frova intubating introducer. Overall, these aspects must be considered on a case by case basis in the context of a markedly diverse and fast changing prehospital environment. Confronted with a patient with significant inhalation injury who is rapidly losing an airway, the practitioner may opt for the superior speed of a stylet to secure it as soon as possible. However, in a scenario where the practitioner is significantly less experienced, the ease-ofuse of a bougie might facilitate success. Thus, where video technology is unavailable, the choice of device might be dictated by a combination of the clinical scenario, practitioner expertise and personal preference. This is in accordance with the results of Sheu et al.'s systematic review and meta-analysis [10].

The issue of practitioner experience has been touched upon and demands further discussion as it is clearly able to significantly influence success with devices assessed in this study. Models of prehospital care and their respective expertise vary across the world, with mainland Europe favouring physician-led services while the UK is predominantly paramedic- and technician-led [29]. Even compared to seemingly similar services such as the USA, there are large differences in personnel competencies, with more comprehensively trained US paramedics recruited at a higher level than in the UK [29]. Therefore, prehospital care systems in different countries may report varying degrees of success with the same array of ETI devices, simply by virtue of their prehospital personnel training and experience with ETI. Participant intubators across our studies varied from physicians, paramedics and emergency medical technicians to even include students. Some of our studies acknowledged and attempted to account for this by recording participants' previous ETI experience and familiarity with the devices being assessed. In line with this, it is integral that pre-existing participant ETI experience is treated as a potential confounding variable in future studies, particularly when the make-up of prehospital care teams has proven to be so varied. 
A mixture of both simulation and real-world prehospital studies were incorporated into our study. We felt that this was necessary to capture a wider range of data due to a lack of real-world prehospital studies, reflecting the distinct challenges involved with research in this setting. A possible solution to this lies with high-quality simulation, perhaps including dedicated advanced prehospital simulation laboratories [30]. By developing high-quality simulation, we can overcome the obstacles of research in the prehospital context (such as lack of control), while still being able to study realistic prehospital scenarios complete with serious game-inspired techniques and methods [30]. This would facilitate the generation of much needed prehospital-oriented randomised controlled trials (RCTs) that can be systematically reviewed to inform guidelines. Without simulation, the power of reviews such as ours becomes handicapped by the methodological quality of the few prehospital studies we can analyse.

It should finally be noted that our study has considered the efficacy of bougies/stylets in the context of their conventional and direct use in facilitating ETI. Two studies were excluded during the selection process that utilised bougies in an exchange manoeuvre, converting from one type of airway to another [31, 32]. One study even used a lighted stylet device for ETI without the aid of a laryngoscope [33]. The efficacy of either of these methods cannot be validated nor revoked by our study. However, readers should have an awareness that the utility of bougies/stylets may not be limited to a singular ETI technique as in this review.

\section{Limitations}

This study is limited in the specific device comparisons that can be made due to the sheer number and variety of bougies/ stylets currently in clinical use or trials. Within the realms of video-assisted ETI alone, variations in software can significantly change a device's performance. Drawing absolute conclusions concerning efficacy has, therefore, proven problematic and general trends and themes have instead been explored. Having such a wide variety of devices available may appear beneficial to prehospital services, though it poses difficulties for synthesis of comprehensive clinical guidelines. This may ultimately lead to ambiguity in clinical decisions regarding what device to use and when. Preexisting experience of participant intubators also limit the conclusions that can be drawn regarding the efficacy of any one device. Aforementioned variations in prehospital provision across the world mean that efficacy of a device tested in the USA may not be comparable to its efficacy in France and vice versa [29]. Future studies must account for this and an internationally standardised method of assessing a prehospital practitioner's ETI capabilities should be employed.
Lastly, this review is limited by a distinct lack of RCTs, with a heavy reliance on observational studies. Sheu et al.'s systematic review and meta-analysis conversely included RCTs only, yet three of their five trials were in a preoperative setting and one was in an ED [10]. This review aimed to focus exclusively on the prehospital efficacy of these devices to guide the practice of prehospital personnel in their unique setting, thus inclusion of hospital-based RCTs was deemed inappropriate. Despite including studies of higher evidence, Sheu et al. concurs in part with the conclusion of our review for when video technology is unavailable, that clinician expertise and personal preference should determine choice of intubating device, owing to a lack of significant differences in primary efficacy outcomes [10]. Nevertheless, the methodological robustness of some of the studies that were included in this review is questionable. Many of the studies ran with admittedly small samples while one study satisfied just $57 \%$ of our quality assessment criteria [23]. This stresses the need for greater quality control amongst prehospital studies, reinstating the importance of high-quality simulation to overcome the inherent lack of controls in a real-world prehospital setting [30].

\section{Conclusions}

It can be concluded that both tracheal tube introducers and stylets function as efficacious aids to intubation in the prehospital environment. However, where video technology is available, bougies could offer a statistically significant advantage in terms of first pass ETI success. The overwhelming variety of devices and competency disparities across different countries' prehospital services make a true assessment of individual efficacy challenging. While acknowledging the potentially higher costs, video-assisted devices may offer distinct advantages and techniques that incorporate this technology could be the answer to further improving ETI success. Where video technology is unavailable, a combination of clinical scenario, practitioner expertise and personal preference might be used to ultimately guide the choice of device. Future studies must account for preexisting practitioner experience when assessing efficacy of any ETI technique and emphasis should be placed on highquality simulation with robust quality control.

Supplementary Information The online version contains supplementary material available at https://doi.org/10.1007/s00068-021-01762-5.

Author contributions Study conception and design were by JT. Material preparation, data collection and analysis were performed by JT and ZA. The first draft of the manuscript was written by JT and both authors commented on previous versions of the manuscript. Both authors read and approved the final manuscript. 
Funding No funding was received to assist with the preparation of this manuscript.

Availability of data Not applicable.

Code availability Not applicable.

\section{Declarations}

Conflict of interest The authors have no conflicts of interest to declare that are relevant to the content of this article.

Ethical approval No ethics approval was needed because this is a systematic review of published studies. No personal, confidential, or sensitive material was collected from the participants in this study. All data used are in the public domain.

Consent to participate There was no need to gain any consent from participants in this review. All participants would have given their consent to be included in the original studies that this review contains, and all the data are now in the public domain.

\section{Consent for publication Not applicable.}

Open Access This article is licensed under a Creative Commons Attribution 4.0 International License, which permits use, sharing, adaptation, distribution and reproduction in any medium or format, as long as you give appropriate credit to the original author(s) and the source, provide a link to the Creative Commons licence, and indicate if changes were made. The images or other third party material in this article are included in the article's Creative Commons licence, unless indicated otherwise in a credit line to the material. If material is not included in the article's Creative Commons licence and your intended use is not permitted by statutory regulation or exceeds the permitted use, you will need to obtain permission directly from the copyright holder. To view a copy of this licence, visit http://creativecommons.org/licenses/by/4.0/.

\section{References}

1. Lockey D, Healey B, Crewdson K, Chalk G, Weaver A, Davies G. Advanced airway management is necessary in prehospital trauma patients. Br J Anaesth. 2015;114(4):657-62.

2. Gnugnoli DM, Singh A, Shafer K. EMS Field Intubation. [Updated 2021 Jan 22]. In: StatPearls [Internet]. Treasure Island (FL): StatPearls Publishing; 2021 Jan-. Available from: https:// www.ncbi.nlm.nih.gov/books/NBK538221/.

3. Crewdson K, Lockey D, Røislien J, Lossius H, Rehn M. The success of pre-hospital tracheal intubation by different pre-hospital providers: a systematic literature review and meta-analysis. Crit Care. 2017;21(1):1.

4. Grape S, Schoettker P. The role of tracheal tube introducers and stylets in current airway management. J Clin Monit Comput. 2016;31(3):531-7.

5. Kerslake D, Oglesby A, Di Rollo N, James E, McKeown D, Ray D. Tracheal intubation in an urban emergency department in Scotland: a prospective, observational study of 3738 intubations. Resuscitation. 2015;89:20-4.

6. Lecky F, Bryden D, Little R, Tong N, Moulton C. Emergency intubation for acutely ill and injured patients. Cochrane Database of Systematic Reviews. 2008.
7. DAS guidelines for management of unanticipated difficult intubation in adults 2015 I Difficult Airway Society [Internet]. Das. uk.com. 2021. Available from: https://das.uk.com/guidelines/das_ intubation_guidelines. Accessed 1 April 2021.

8. Apfelbaum J, Hagberg C, Caplan R, Blitt C, Connis R, Nickinovich $\mathrm{D}$, et al. Practice guidelines for management of the difficult airway. Anesthesiology. 2013;118(2):251-70.

9. Guidelines: Prehospital resuscitation [Internet]. Resuscitation Council UK. 2021. Available from: https://www.resus.org.uk/ library/2015-resuscitation-guidelines/prehospital-resuscitation. Accessed 1 April 2021.

10. Sheu Y, Yu S, Huang T, Liu F, Lin Y, Tam K. Comparison of the efficacy of a bougie and stylet in patients with endotracheal intubation: a meta-analysis of randomized controlled trials. J Trauma Acute Care Surg. 2019;86(5):902-8.

11. Moher D, Liberati A, Tetzlaff J, Altman D. Preferred reporting items for systematic reviews and meta-analyses: the PRISMA statement. BMJ. 2009;339(jul21 1):b2535-b2535.

12. Study Quality Assessment Tools I NHLBI, NIH [Internet]. Nhlbi.nih.gov. 2021 [cited 1 April 2021]. Available from: https://www.nhlbi.nih.gov/health-topics/study-quality-asses sment-tools. Accessed 1 April 2021.

13. DerSimonian R, Laird N. Meta-analysis in clinical trials. Control Clin Trials. 1986;7(3):177-88.

14. Bonnette A, Aufderheide T, Jarvis J, Lesnick J, Nichol G, Carlson J, et al. Bougie-assisted endotracheal intubation in the pragmatic airway resuscitation trial. Resuscitation. 2021;158:215-9.

15. Ångerman S, Kirves H, Nurmi J. A before-and-after observational study of a protocol for use of the C-MAC videolaryngoscope with a Frova introducer in pre-hospital rapid sequence intubation. Anaesthesia. 2018;73(3):348-55.

16. Jabre P, Combes X, Leroux B, Aaron E, Auger H, Margenet A, et al. Use of gum elastic bougie for prehospital difficult intubation. Am J Emerg Med. 2005;23(4):552-5.

17. Karaca O, Bayram B, Oray N, Acerer A, Sofuoglu Z. Comparison of the airway access skills of prehospital staff in moving and stationary ambulance simulation: a randomized crossover study. Turk J Emerg Med. 2017;17(2):35-41.

18. Phelan M, Moscati R, D'Aprix T, Miller G. Paramedic use of the endotracheal tube introducer in a difficult airway model. Prehosp Emerg Care. 2003;7(2):244-6.

19. Cooney D, Beaudette C, Clemency B, Tanski C, Wojcik S. Endotracheal intubation with a video-assisted semi-rigid fiberoptic stylet by prehospital providers. Int J Emerg Med. 2014;7(1):1

20. Heegaard W, Black C, Pasquerella C, Miner J. Use of the endotracheal tube introducer as an adjunct for oral tracheal intubation in the prehospital setting. AirMed. 2003;22(1):28-31.

21. Combes X, Jabre P, Margenet A, Merle J, Leroux B, Dru M, et al Unanticipated difficult airway management in the prehospital emergency setting. Anesthesiology. 2011;114(1):105-10.

22. Woollard M, Lighton D, Mannion W, Watt J, McCrea C, Johns I, et al. Airtraq vs standard laryngoscopy by student paramedics and experienced prehospital laryngoscopists managing a model of difficult intubation. Anaesthesia. 2007;63(1):26-31.

23. Messa M, Kupas D, Dunham D. Comparison of bougie-assisted intubation with traditional endotracheal intubation in a simulated difficult airway. Prehosp Emerg Care. 2011;15(1):30-3.

24. Evrin T, Smereka J, Gorczyca D, Bialka S, Ladny J, Katipoglu $\mathrm{B}$, et al. Comparison of different intubation methods in difficult airways during simulated cardiopulmonary resuscitation with continuous chest compression: a randomized cross-over manikin trial. Emerg Med Int. 2019;2019:1-7.

25. Gregory P, Woollard M, Lighton D, Munro G, Jenkinson E, Newcombe R, et al. Comparison of malleable stylet and reusable and 
disposable bougies by paramedics in a simulated difficult intubation. Anaesthesia. 2012;67(4):371-6.

26. Le D, Reed D, Weinstein G, Gregory M, Brown L. Paramedic use of endotracheal tube introducers for the difficult airway. Prehosp Emerg Care. 2001;5(2):155-8.

27. De Jong A, Molinari N, Conseil M, Coisel Y, Pouzeratte Y, Belafia F, et al. Video laryngoscopy versus direct laryngoscopy for orotracheal intubation in the intensive care unit: a systematic review and meta-analysis. Intensive Care Med. 2014;40:629.

28. Howard-Quijano K, Huang Y, Matevosian R, Kaplan M, Steadman R. Video-assisted instruction improves the success rate for tracheal intubation by novices. Br J Anaesth. 2008;101(4):568-72.

29. Lockey D, Deakin C. Pre-hospital trauma care: systems and delivery. Contin Edu Anaesth Crit Care Pain. 2005;5(6):191-4.
30. Maurin Söderholm H, Andersson H, Andersson Hagiwara M, Backlund P, Bergman J, Lundberg L, et al. Research challenges in prehospital care: the need for a simulation-based prehospital research laboratory. Adv Simul. 2019;4(1):1.

31. Beleña J, Gasco C, Polo C, Vidal A, Núñez M, Lopez-Timoneda F. Laryngeal mask, laryngeal tube, and frova introducer in simulated difficult airway. J Emerg Med. 2015;48(2):254-9.

32. Lutes M, Worman D. An unanticipated complication of a novel approach to airway management. J Emerg Med. 2010;38(2):222-4.

33. Vollmer T, Stewart R, Paris P, Ellis D, Berkebile P. Use of a lighted stylet for guided orotracheal intubation in the prehospital setting. Ann Emerg Med. 1985;14(4):324-8. 